Parasites in the immunized host: mechanisms of survival

Ciba Foundation Symposium no. 25 (new series). 1974. Amsterdam: Associated Scientific Publishers. Pp. viii and 280. US \$16.20.

Vaccination against many important helminth and protozoan parasites of man (and of domestic animals) will probably become possible only if methods are developed to produce better immunisation than occurs after natural infection; as one contributor to this symposium put it, we must aim to " improve on nature". One important element of induced resistance must be to counter the escape mechanisms employed by parasites that allow them to survive in the immunised host, and this book is the product of a symposium concerned with a detailed review of these mechanisms.

The book contains 13 chapters, which were the working papers for the symposium, together with the extensive and carefully edited discussion on each topic-certainly as valuable a part of the book as the formal presentations. There is, first, a brief and general review of immune responses to parasites, followed by four chapters on antigenic variation. These cover the genetics of antigenic variation in the free-living protozoan Paramecium and consideration of the extent to which some of the observations made might be applicable to parasitic protozoa; the induction of and immune response to intra-strain variants in malaria; antigenic variation in trypanosomes, and the nature of the antigens and their expression; and survival of the rat nematode Nippostrongylus brasiliensis in an immune host and comparison of " adapted" and normal worms.

The next chapter deals with variations in immunological responsiveness that leads to a spectrum of clinical manifestations of disease, e.g., in leprosy, leishmaniasis, syphilis and Chagas' disease. There are two chapters concerned with depression of the immune response during protozoal infections, which deal in particular with experiments on murine malaria and with tests of the immune competence of patients with malaria or trypanosomiasis. Soluble antigens are released during malarial and other protozoan infections, and the various ways in which these could interfere with the immune response are considered. A detailed account is given of concomitant immunity in schistosome infections and of the host-like antigenic determinants expressed on the surface membranes of the adult flukes, and there is also a description of observations indicating two types of specific suppression of immunopathological changes in schistosomiasis that reduce the harmful effects of infection. Finally, there are two chapters dealing with survival of the intracellular parasites Toxoplasma and Leishmania, which are found within macrophages of the host.

The last chapter is a summary of the conclusions of the symposium on the ways in which parasites appear to escape the host response, and the implications of this for prophylaxis. Altogether this is a most useful book. Immunological texts on parasitic infections are all too often rather diffuse because they attempt to cover too many aspects of the subject. It is pleasing, therefore, to have a text produced to a very high standard which deals in detail with one (fascinating) aspect of immunity to worm and protozoan parasites. The book is recommended without reservation because there is much here to stimulate both the specialist and the reader with only a general interest in this subject.

G. A. T. TARGETT

\title{
Mononuclear phagocytes in immunity, infection and pathology
}

Edited by R. van Furth. 1975. Oxford: Blackwell Scientific Publications. Pp. xviii and 1062. $£ 32 \cdot 50$.

The publication of the proceedings of a symposium approximately 2 years afterwards and at a cost of $£ 1$ for every 33 pages raises questions about the objectives and methods of scientific communication. The present book on the proceedings of the Second Conference on Mononuclear Phagocytes held at Leiden gives a good account of the knowledge of macrophages in 1973. There are sections on structure, origin, phagocytosis, cell-surface characteristics, biochemistry, phagosome-lysosome interaction, cell-mediated immunity and resistance to infection, antibody formation, tumour immunity and granuloma formation. 